\title{
In vivo continuous monitoring of L-lactate coupling subcutaneous microdialysis and an electrochemical biocell
}

\author{
G. Volpe ${ }^{\text {a }}$, D. Moscone ${ }^{\text {a }}$, D. Compagnone ${ }^{\text {a }}$, G. Palleschi ${ }^{b, *}$ \\ a Diparimento di Scienze e Tecnologie Chimiche, Università di Roma Tor Vengata', via della Ricerca Scientifica, D0133 Rome, Italy \\ b Dipartimento di Scienze degli Alimenti, Università di Napoli 'Federico II', via Università 100, 80055 Portici, Italy
}

\begin{abstract}
The microdialysis technique and a flow-through cell assembled with a lactate amperometric sensor have been coupled for continuous monitoring of lactate in subcutaneous tissue. Different fiber lengths and different flow rates have been tested to optimize the performance of the analytical system. The lactate probe is prepared by placing a nylon net membrane with immobilized lactate oxidase onto the platinum electrode inserted into the cell. Probe stability and reproducibility have been optimized. The response time of the biosensor is less than two minutes. In vivo experiments based on four hour continuous lactate monitoring on a rabbit showed good sensor stability and reproducibility. The same experiments on human volunteers showed a lactate increase during physical exercise. Compared with a reference spectrophotometric procedure, the results correlated well.
\end{abstract}

Keywords: Electrochemical biocell; In vivo monitoring; L-lactatc; Subcutancous microdialysis

\section{Introduction}

Lactate, an intermediate metabolite of anacrobic glycolysis, is a substrate that has long been of interest in physiology and clinical medicine. Increased concentrations of blood lactate are observed during physical exercise, and changes in these concentrations are useful for estimating the intensity of the exercise [1]. Lactate is a metabolite that it is important to monitor rapidly in people who are sick enough to be in special-care units in hospital, particularly cardiac patients [2]. Lactate monitors are also used in diabetes control, and in sport medicine to help athletes optimize their training [3]. Lactate determination is usually assessed by chemical analysis ex vivo being collected by invasive techniques that in most cases do not reflect the fate and physiology of this metabolite [4].

Microdialysis is an in vivo sampling technique that continuously removes small quantities of metabolites from the fluid compartments of the body, such as brain tissue, the bloodstream, subcutaneous fluid, etc, without removing any liquid $[5,6]$.

If the dialysis tube is a microtube with a diameter of $100-200 \mu \mathrm{m}$ and if the flow is very low, the amount

\footnotetext{
* Corresponding author.
}

taken out does not alter the concentration of the tissue fluid [7-9].

For continuous monitoring of lactate in vivo, we coupled the microdialysis technique with a lactate biosensor. We inserted, in subcutaneous tissue, a hollow microdialysis fibre that is perfused with a physiological buffer at a constant flow rate. The dialysate flows continuously into a cell provided with a lactate biosensor based on the enzyme lactate oxidase immobilized on a polymer support. In this paper we present the analytical results of the lactate probe during microdialysis and for in vivo experiments on a rabbit and human volunteers.

\section{Materials and methods}

\subsection{Materials}

Lactate oxidasc EC1.1.3.2., from Pediococcus sp., 39

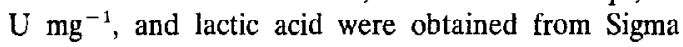
Chemical Co., St. Louis, MO, USA. This enzyme was immobilized on a nylon net as previously reported [10].

Dulbecco's physiological buffer ( $\mathrm{pH}$ 7.4) was prepared in doubly distilled water. All chemicals used were of analytical grade. 
Cellulose acetate $(53 \%$ acetyl) and polyvinyl acetate of high molecular weight were obtained from Farmitalia Carlo Erba (Milan, Italy). For casting the cellulose acetate membrane a precision gauge tool (from Precision Gage and Tool $\mathrm{Co}$, Dayton, $\mathrm{OH}$ ) was used. This membrane, with about $100 \mathrm{Da}$ MWCO (molecular weight cut off), was prepared as previously reported [11-13].

A peristaltic pump, Minipuls 3, for flow analysis was from Gilson (France). A Rheodyne valve (USA), model 7125 , was used as the injection valve. Alternatively, a CMA/160 On-line Injector automatically activated by a CMA/100 Microinjection Pump (CMA/Microdialysis, Stockholm, Sweden) was used.

A wall-jet cell was obtaincd from Metrohm (model 656 Electrochemical Detector, Herisau, Switzerland). However, the original working electrode was replaced with a platinum electrode, with a diameter of $1.6 \mathrm{~mm}$, obtained from BAS (model MF 2013). This cell was connected to an Amperometric Biosensor Detector (ABD) from Universal Sensors Inc., Metairie, LA. The current output was recorded with a $\mathrm{L} 6512 x-t$ recorder (Linseis, Selb, German).

To assemble the microdialysis probe we used regenerated cellulose Spectra/Por Hollow fiber (internal diameter $215 \mu \mathrm{m}$, wall thickness $18 \mu \mathrm{m}$, MWCO 6000 $\mathrm{Da}$, obtained from Spectrum Medical Industries Inc, Los Angeles, CA). We insert into the hollow fiber a gold-plated tungsten wire of $50 \mu \mathrm{m}$ diameter obtained from Goodfellow, Cambridge, UK.

Silicone tubing, internal diameter $0.3 \mathrm{~mm}$, outer diameter $0.63 \mathrm{~mm}$, wall thickness $0.165 \mathrm{~mm}$, form A$\mathrm{M}$ Systems and nylon tubing internal diameter 0.25 $\mathrm{mm}$, outer diameter $0.75 \mathrm{~mm}$, wall thickness $0.25 \mathrm{~mm}$, from Firie (Genoa, Italy) were used to connect the hollow fibers to the flow system.

Spectrophotometric measurements were carried out with a Unicam $8625 \mathrm{UV} / \mathrm{Vis}$ spectrophotometer (Italy) using a Boehringer kit.

\subsection{Assembly of the sensor}

The wall-jet cell used in this work consists of a platinum electrode covered with the cellulose acetate membrane, which protects the electrode surface from electrochemical interferences such as ascorbate and urate. The enzymatic membrane is placed in the middle and an outer membrane (cellulose acetate, 15000 MWCO) is used. A small piece of Teflon tube of a suitable diameter is used to stretch the membranes on the $\mathrm{Pt}$ electrode surface.

The cell assembly is completed by a silver-silver chloride cathode and a platinum auxiliary electrode.

\subsection{Procedure}

The procedure is the following: lactate, collected by the microdialysis probe, passes through the cell and

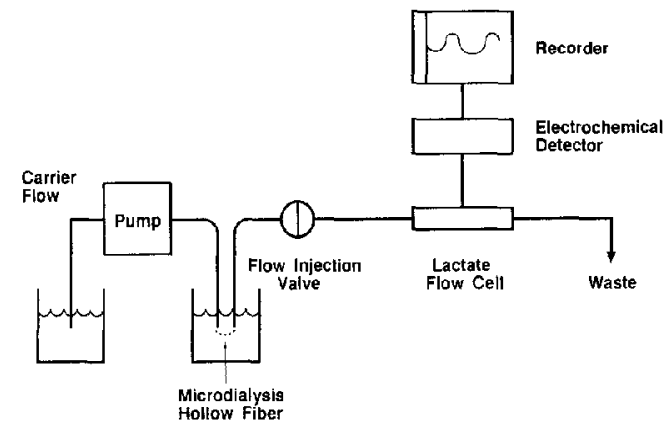

Fig. 1. Scheme of the flow system for in vitro and in vivo experiments.

reacts with the enzyme lactate oxidase according to the following reaction:

Lactate $+\mathrm{O}_{2}+\mathrm{H}_{2} \mathrm{O} \longrightarrow$ Pyruvate $+\mathrm{H}_{2} \mathrm{O}_{2}$

The hydrogen peroxide produced is oxidized at the platinum electrode, which is a part of a wall-jet cell assembled with a three-electrode configuration. The anodic current produced is correlated to the concentration of lactate in the dialysate.

The flow system for in vitro experiments is shown in Fig. 1. The peristaltic pump drives the carrier solution, at constant rate, through the microdialysis probe immersed in lactate standards and a steady-state current is measured. During experiments the lactate standard solutions in which the microdialysis probe was immersed were changed manually. In vivo experiments have been carried out using sterilized hollow fibers.

To place the microdialysis hollow fiber subcutaneously, a sterilized needle was inserted transcutaneously and the needle tip was pulled out. Then the sterilized fiber was inserted through the needle tip and the needle was taken out, leaving the hollow fiber under the skin. The fiber was connected to Teflon tubes and fixed with epoxy glue.

For checking variations in sensitivity during the experiments, a flow-injection system was used. A Rheodyne injection valve with a $20 \mu$ sample loop was introduced into the flow system just after the microdialysis probe.

\section{Results and discussion}

\subsection{In vitro experiments}

The lactate profile measured with the lactate flow cell, without the microdialysis probe, at different flow rates is shown in Fig. 2. In this case and during the analytical evaluation of the lactate biocell, we did not use the outer dialysis membrane so we obtained the highest probe sensitivity and the fastest response time. The flow rate was varied from 5 to $100 \mu \mathrm{m} \mathrm{min}^{-1}$. When the flow rate was increased, the lactate response decreased. This phenomenon was more evident from 


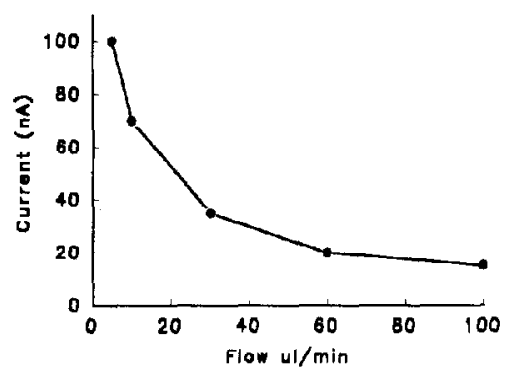

Fig. 2. Lactate profile with the lactate flow cell assembled with cellulose acetate and nylon net.

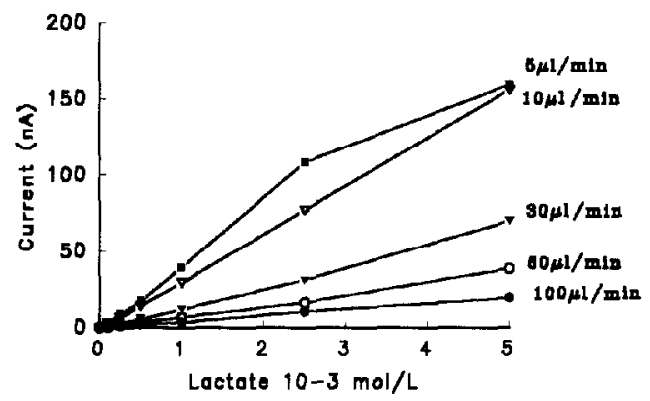

Fig. 3. Calibration curves of the lactate biocell coupled with a microdialysis probe at different flow rates.

5 to $30 \mu 1 \mathrm{~min}^{-1}$ and levelled-off from 60 to $100 \mu \mathrm{l}$ $\min ^{-1}$.

Fig. 3 reports some calibration curves of the lactate biocell coupled with a microdialysis probe using different flow rates. Experiments carried out at flow rates of 5-10 $\mu \mathrm{l} \mathrm{min}{ }^{-1}$ showed a higher response. However, at these flow rates the response time was longer, so the best compromise was reached using a flow rate of 30 $\mu 1 \mathrm{~min}^{-1}$. Fig. 4(a) shows some calibration curves of lactate using different microdialysis fiber lengths.

During this experiment the flow rate was kept constant at $30 \mu \mathrm{min}^{-1}$. As is seen from this Figure, the longer the fiber is, the higher is the response. However, longer fibers mean more lactate recovery from the interstitial space of the subcutaneous tissuc, which can alter the tissue fluid. Fig. 4(b) shows the percentage recovery of lactate using different fiber lengths. Results are the mean of three different concentrations of lactate standards $\left(0.1,0.25,0.5 \mathrm{mmol}^{-1}\right)$.

Also in this case a compromise was reached by selecting a fiber of $2 \mathrm{~cm}$ length.

As stated previously, all these experiments were carried out with the lactate probe assembled with a cellulose acetate membrane and the enzyme membrane in direct contact with the analysing solution. However, we observed a decrease in probe sensitivity of $20 \%$ in $12 \mathrm{~h}$ of continuous monitoring.
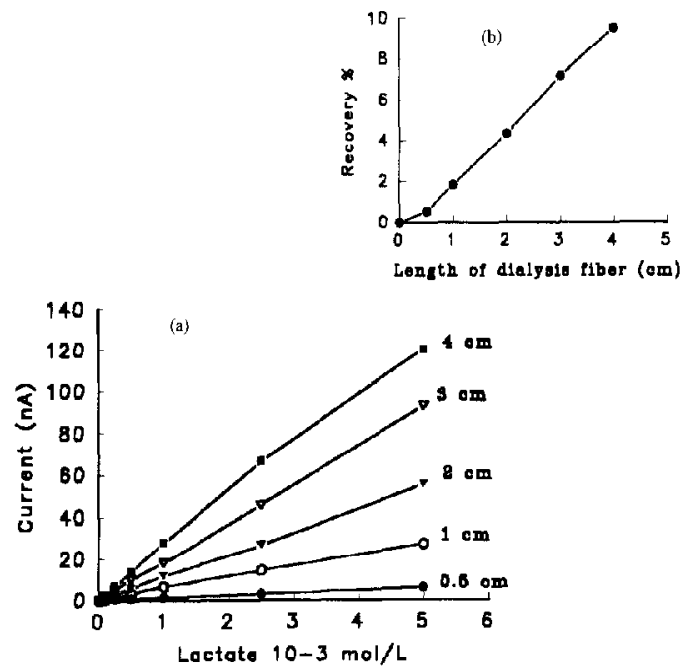

Fig. 4. (a) Calibration curves of lactate using different microdialysis fiber lengths. (b) Lactate recovery obtained by varying the length of the dialysis fiber.

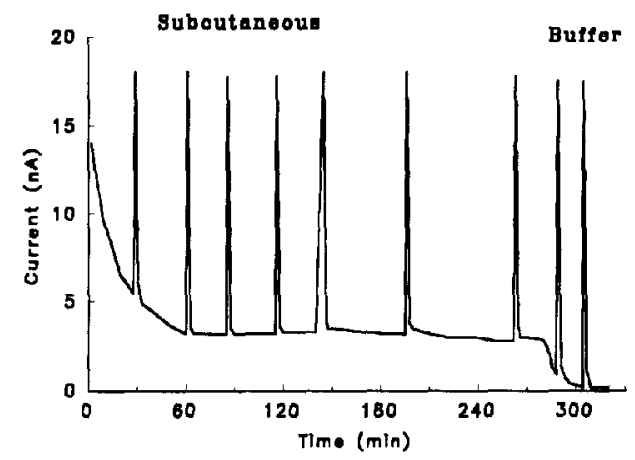

Fig. 5. Lactate experiment in a rabbit using a subcutaneous hollow fiber. Spikes represent a lactate standard of $10^{-4} \mathrm{~mol}^{-1}$.

To avoid this problem, we covered our probe with a dialysis membrane of $15000 \mathrm{MWCO}$. In this way, the enzyme being more protected, it did not show any decrease in sensitivity with time. We therefore decided to use this assembly for further experiments.

\subsection{In vivo experiments}

In vivo experiments were carried out first on an awake rabbit, where a four-hour continuous monitoring of lactate in subcutaneous tissue showed a rather stable signal and good sensitivity of the biosensor. 'I he biosensor sensitivity was checked by the flow-injection valve located in the flow stream of the microdialysis probe (see Fig. 5). A lactate standard buffered solution filled the loop of the injection valve and flowed through the lactate biosensor. We obtained a current profile similar to a peak. 
Then the hollow fiber was inserted in the subcutaneous tissue of three volunteers and the same experiment as carried out on a rabbit was performed, but the protocol was changed. In the first experiment reported in Fig. 6 , the subject performed different exercises which in all cases resulted in a lactate increase, this increase depending of the intensity and length of the exercise.

In the second experiment report in Fig. 7, a lactate profile is shown for a subject who had a meal and then performed physical exercise. The meal caused a moderate increase of lactate, but when the subject performed physical exercise the lactate increased markedly. After this point, the microdialysis probe broke and no more lactate was collected by the fiber, so the experiment was interrupted. The third experiment shows a lactate profile of a subject who was asked to perform the same physical exercises for different periods of time. As is seen from Fig. 8, the lactate increase reflects the length of the exercise performed.

Dots represent the lactate measured with a spectrophotometric procedure. To correlate the continuous monitoring of the current output with the lactate value, we assume that the current value before the meal or the exercise corresponds to the lactate value of the first (Fig. 7) and the second (Fig. 8) blood sample (one-point calibration), respectively.

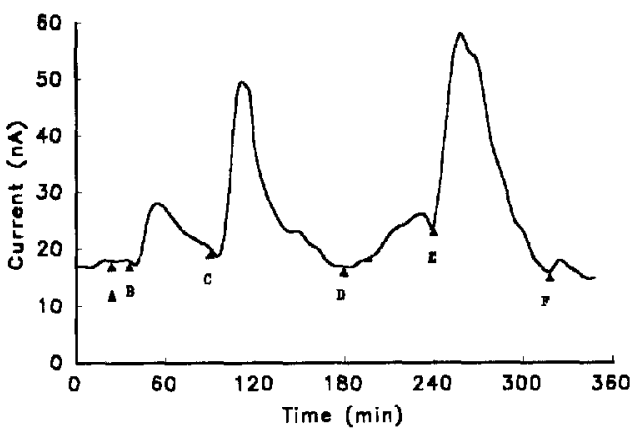

Fig. 6. Lactate profile during a subcutaneous experiment: A, B, C, $D, E, F$ represent times when different exercises were performed.

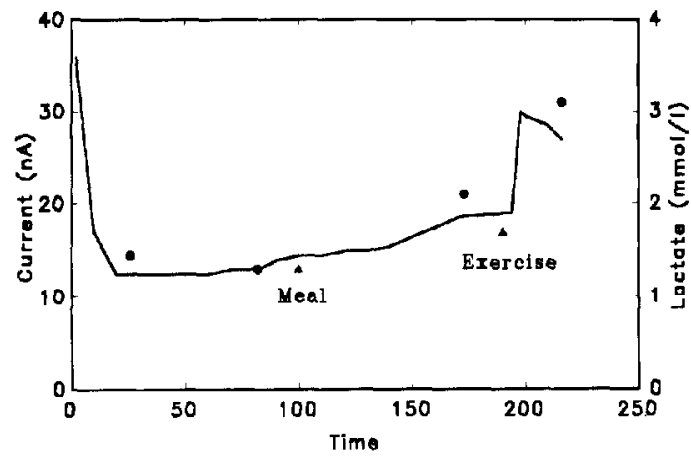

Fig. 7. Lactate profile during a subcutaneous experiment compared with a spectrophotometric procedure (solid dots).

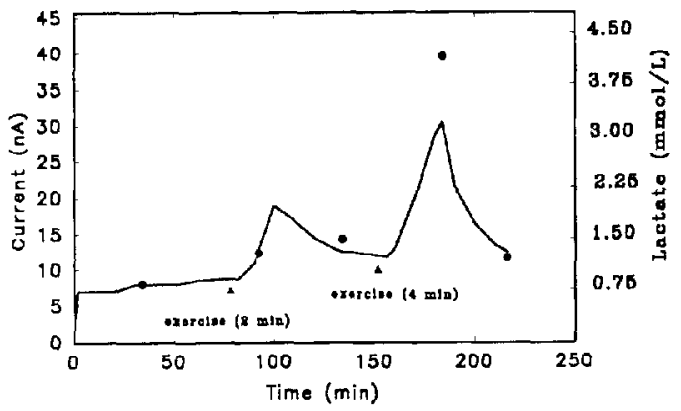

Fig. 8. Lactate profile during a subcutaneous experiment compared with a spectrophotometric procedure (solid dots).

\section{Acknowledgement}

This research was supported by the CNR Target Project Biotechnology and Bioinstrumentation.

\section{References}

[1] J. Karlson, Muscle exercise, energy metabolism and blood lactate, Adv. Cardiol., 35 (1986) 35-46.

(2) R.T. Smolenski, J. Swicrczynski, M. Narkiewicz and M.M. Zydowo, Purines, lactate and phosphate release from child and adult heart during cardioplegic arrest, Clin. Chim. Acta, 192 (1990) 155-164.

[3] N. Silprandi, Aspetti biochimici dell'esercizio fisico e dell'allenamento, Biochim. Clin., 12 (1988) 165-170.

[4] M. Mascini, S. Fortunati, G. Palleschi, M. Massi-Benedetti and P. Fabietti, An L-lactate sensor with immobilized enzyme for use in vivo studies with an endocrine artificial pancreas, Clin. Chem., 31 (1985) 451-453.

[5] P.K. Kissinger, Biomedical applications of liquid chromatography-electrochemistry, J. Chromatogr., 488 (1989) 31.

[6] Y.L. Hurd, J. Kehr and U. Ungerstedt, In vivo microdialysis as a technique to monitor drug transport: correlation of extracellular cocaine levels and dopamine nverflow in the rat brain, J. Neurochem., 51 (1988) 1314.

[7] D. Moscone, M. Pasini and M. Mascini, Subcutaneous microdialysis probe coupled with glucose biosensor for in vivo continuous monitoring, Talanta, 8 (1992) 1039-1044.

[8] D. Moscone and M. Mascini, Microdialysis and glucose biosensor for in vivo monitoring, Ann. Biol. Clin., 50 (1992) 323.

[9] D. Mosconc and M. Mascini, In vivo monitoring with microdialysis probe, in G.G. Guilbault and M. Mascini (eds.), Uses of Immobilized Biological Compounds, Kluwer, Dordrecht, 1993, pp. 115-122.

[10] M. Mascini, G. Palleshi and M. lannello, Enzyme electrodes with improved mechanical and analytical characteristics obtained by binding enzymes to nylon nets, Anal. Chim. Acta, 156 (1983) 135.

[11] M. Mascini and F. Mazzei, Amperometric sensor for pyruvate with immobilized pyruvate oxidase, Anal. Chim. Acta, 192 (1987) 9.

[12] P.J. Taylor, E. Kmetec and J.M. Johnson, Design, construction and applications of a galactose selective electrode, Anal. Chem., 49 (1977) 798.

[13] G. Palleschi, M.A. Nabi Rahni, G.J. Lubrano, J.N. Ngwainbi and G.G. Guilbault, A study of interferences in glucose measurements in blood by hydrogen peroxide based glucose probes, Anal. Biochem., 159 (1986) 144. 